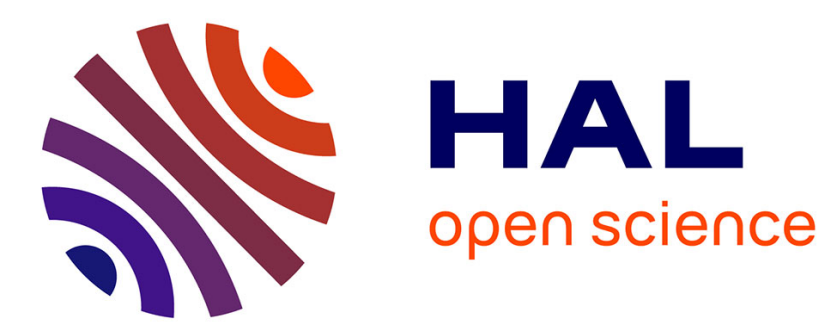

\title{
Phase-amplitude coupling characteristics in directly modulated quantum dot lasers
}

Cheng Wang, M Osiński, J Even, F Grillot

\section{To cite this version:}

Cheng Wang, M Osiński, J Even, F Grillot. Phase-amplitude coupling characteristics in directly modulated quantum dot lasers. Applied Physics Letters, 2014, 105 (22), pp.233103 - 21105. 10.1063/1.4903493 . hal-01091745

\section{HAL Id: hal-01091745 \\ https://hal.science/hal-01091745}

Submitted on 8 Dec 2014

HAL is a multi-disciplinary open access archive for the deposit and dissemination of scientific research documents, whether they are published or not. The documents may come from teaching and research institutions in France or abroad, or from public or private research centers.
L'archive ouverte pluridisciplinaire HAL, est destinée au dépôt et à la diffusion de documents scientifiques de niveau recherche, publiés ou non, émanant des établissements d'enseignement et de recherche français ou étrangers, des laboratoires publics ou privés. 


\section{AIP Applied Physics Letters}

\section{Phase-amplitude coupling characteristics in directly modulated quantum dot lasers}

C. Wang, M. Osiński, J. Even, and F. Grillot

Citation: Applied Physics Letters 105, 221114 (2014); doi: 10.1063/1.4903493

View online: http://dx.doi.org/10.1063/1.4903493

View Table of Contents: http://scitation.aip.org/content/aip/journal/apl/105/22?ver=pdfcov

Published by the AIP Publishing

\section{Articles you may be interested in}

Gain compression and its dependence on output power in quantum dot lasers

J. Appl. Phys. 113, 233103 (2013); 10.1063/1.4811458

High performance laser linewidth broadening for stimulated Brillouin suppression with zero parasitic amplitude modulation

J. Appl. Phys. 105, 093104 (2009); 10.1063/1.3116149

Frequency and amplitude modulation in terahertz-sideband generation in quantum wells

Appl. Phys. Lett. 94, 021105 (2009); 10.1063/1.3068494

High-resolution gas phase spectroscopy with a distributed feedback terahertz quantum cascade laser Appl. Phys. Lett. 89, 061115 (2006); 10.1063/1.2335803

Improved laser modulation response by frequency modulation to amplitude modulation conversion in transmission through a fiber grating

Appl. Phys. Lett. 71, 879 (1997); 10.1063/1.119676

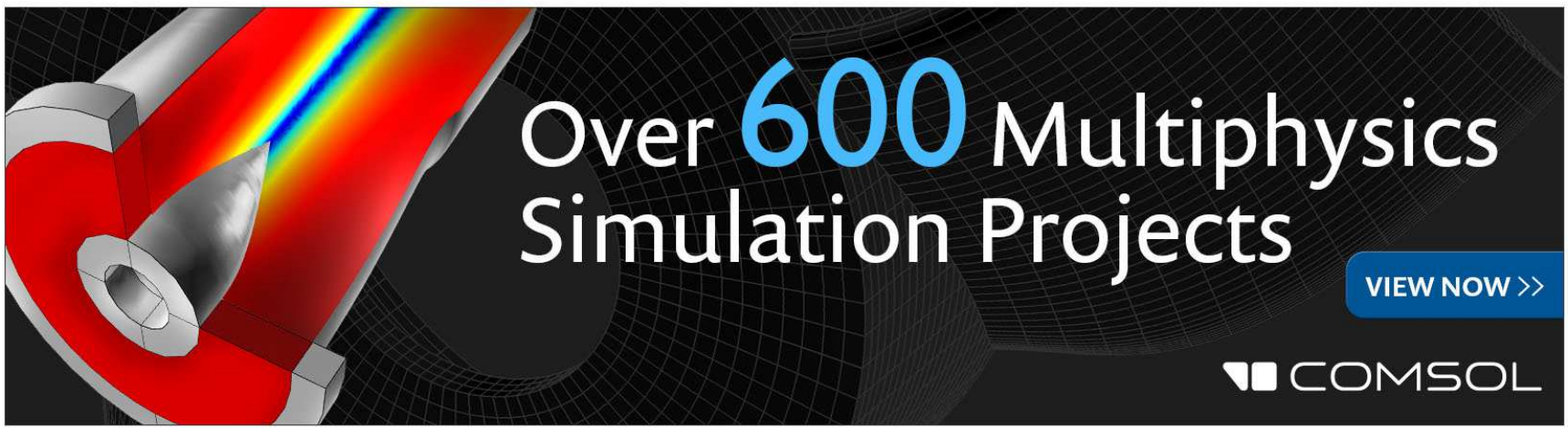




\title{
Phase-amplitude coupling characteristics in directly modulated quantum dot lasers
}

\author{
C. Wang, ${ }^{1,2}$ M. Osiński, ${ }^{1,3}$ J. Even, ${ }^{2}$ and F. Grillot ${ }^{1}$ \\ ${ }^{1}$ Télécom ParisTech, Ecole Nationale Supérieure des Télécommunications, CNRS LTCI, \\ 75634 Paris Cedex 13, France \\ ${ }^{2}$ Institut National des Sciences Appliquées, Université Européenne de Bretagne, 35708 Rennes Cedex 7, France \\ ${ }^{3}$ Center for High Technology Materials, University of New Mexico, Albuquerque, \\ New Mexico 87106-4343, USA
}

(Received 30 October 2014; accepted 23 November 2014; published online 4 December 2014)

\begin{abstract}
We present a semi-analytical model for studying the phase-amplitude coupling ( $\alpha$-factor) in quantum dot (QD) semiconductor lasers, which takes into account the influence of carrier populations in the excited state and in the two-dimensional carrier reservoir on the refractive index change. Calculations of the $\alpha$-factor based on the amplified spontaneous emission method and on the "FM/AM" technique are both investigated. It is shown that the $\alpha$-factor of a QD laser strongly depends on the energy separation between the ground state and the off-resonant states. Through band structure engineering, the $\alpha$-factor can be reduced by enlarging this energy separation. (C 2014 AIP Publishing LLC.
\end{abstract}

[http://dx.doi.org/10.1063/1.4903493]

The linewidth enhancement factor ( $\alpha$-factor) of semiconductor lasers plays a crucial role in driving fundamental features, such as the spectral linewidth broadening, frequency chirp, and mode stability, as well as nonlinear dynamics under optical injection or optical feedback. ${ }^{1-3}$ It describes the amplitude-phase coupling of the electric field as well as the coupling between the carrier-induced refractive index and the gain variations in semiconductor lasers, which is characterized by the complex optical susceptibility $\left(\chi=\chi^{\prime}+j \chi^{\prime \prime}\right)$ as $^{4}$

$$
\alpha_{H}=\frac{\partial \chi^{\prime} / \partial N}{\partial \chi^{\prime \prime} / \partial N}
$$

where $\mathrm{N}$ stands for the carrier density.

Quantum-well (QW) lasers commonly exhibit $\alpha$-factor values in the range of $2.0-5.0 .^{5}$ In contrast, the reported $\alpha$-factor in quantum-dot (QD) lasers spread over a wide range from zero up to more than $10 .^{6-9}$ This large discrepancy can be attributed to the complex carrier dynamics in the QD laser. Figure 1 shows a sketch of the gain spectrum in a QD laser. The gain for laser emission mainly arises from the carrier population in the resonant ground state $(\mathrm{GS}){ }^{10,11} \mathrm{In}$ contrast, populations in the off-resonant excited states (ES) and the 2D carrier reservoir (RS) significantly change the symmetry of the gain spectrum and thus vary the refractive index through the Kramers-Kronig relation. ${ }^{10-12}$ In addition, the free-carrier plasma effect also plays an important role in the refractive index variation. ${ }^{13,14}$

This work proposes an improved rate equation model taking into account the contribution of off-resonant states to the refractive index change, which allows semi-analytical study of the QD laser's $\alpha$-factor as well as the exploration of underlying physical mechanisms. In a semi-classical theory, the semiconductor laser system can be fully described by the optical Bloch equations together with the Maxwell's equations. ${ }^{15}$ To simplify the model, this work treats the electrons and holes as neutral pairs (excitons). The charged carriers are assumed to be directly injected from the continuum RS into the dots. In the dot, the discrete GS and the first ES are taken into account. Neglecting the inhomogeneous broadening effect, the slowly varying complex electric field amplitude $E(t)$ is given by: ${ }^{16}$

$$
\frac{d}{d t} E(t)=\frac{j \omega_{L S}^{0} \Gamma_{P}}{2 \varepsilon_{b g} \varepsilon_{0}} \frac{1}{A_{R S} H_{B}} \sum_{X=G S, E S, R S}\left(\mu_{X}^{*} P_{X}\right)-\frac{1}{2 \tau_{P}} E(t),
$$

where $\omega_{L S}^{0}$ is the lasing frequency in the cold cavity, $\varepsilon_{0}$ and $\varepsilon_{b g}$ are the vacuum permittivity and the relative permittivity of the background material, respectively. $\Gamma_{P}$ is the optical confinement factor, $A_{R S}$ is the surface area of the RS, and $H_{B}$ is the height which is considered the same as the dot's height in this work. ${ }^{17}$ The sum over $\mathrm{X}(\mathrm{X}=\mathrm{GS}, \mathrm{ES}, \mathrm{RS})$ includes all possible optical transitions, with $\mu_{X}$ being the corresponding dipole transition matrix element and $P_{X}$ being the microscopic polarization. $\tau_{P}$ is the photon lifetime in the laser cavity. Through the relation between the electric field and the polarization, we obtain the complex gain: ${ }^{16}$

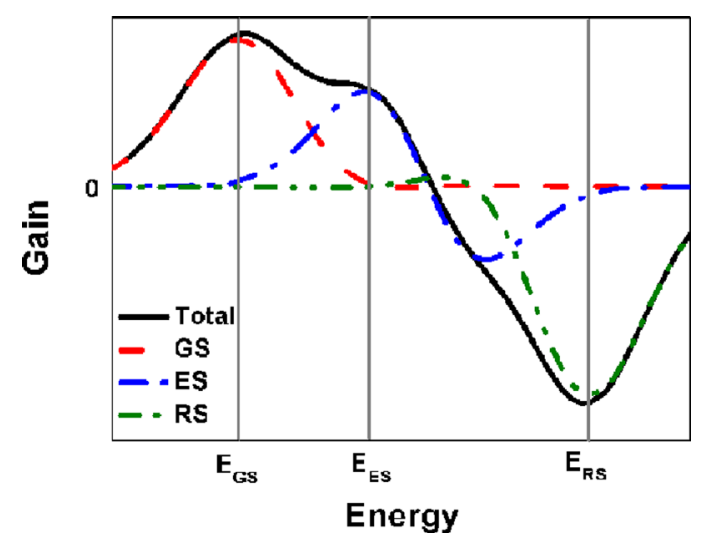

FIG. 1. An illustration of the gain spectrum in a QD laser, including the resonant GS, the off-resonant ES and the RS. 


$$
\begin{aligned}
\tilde{G}\left(\omega_{L S}^{0}, t\right)= & \left(2 \mu_{G S}^{*} \frac{j \omega_{L S}^{0} \Gamma_{P}}{2 \varepsilon_{0} \varepsilon_{b g}} \frac{2 N_{B}}{H_{B}} \frac{P_{G S}(t)}{E(t)}\right) \\
& +\left(4 \mu_{E S}^{*} \frac{j \omega_{L S}^{0} \Gamma_{P}}{2 \varepsilon_{0} \varepsilon_{b g}} \frac{2 N_{B}}{H_{B}} \frac{P_{E S}(t)}{E(t)}\right) \\
& +\left(\frac{j \omega_{L S}^{0} \Gamma_{P}}{2 \varepsilon_{0} \varepsilon_{b g}} \frac{2}{A_{R S} H_{B}} D_{R S} \mu_{R S}^{*} \frac{P_{R S}(t)}{E(t)}\right),
\end{aligned}
$$

where the RS is treated as a discrete energy state of degeneracy $\mathrm{D}_{\mathrm{RS}},{ }^{18}$ and $\mathrm{N}_{\mathrm{B}}$ is the QD density per unit area. The real part of Eq. (3) is related to the laser gain, while the imaginary part gives the instantaneous frequency shift of the electric field. The three terms on the right hand give contributions of the GS, the ES and the RS, respectively. Introducing the differential gain $\left(a_{X}\right),{ }^{19}$ the material gain of each state is given by:

$$
\begin{aligned}
g_{G S} & =\frac{a_{G S}}{1+\xi S_{G S}} \frac{N_{B}}{H_{B}}\left(\frac{2 N_{G S}}{2 N_{B} / H_{B}}-1\right), \\
g_{E S} & =a_{E S} \frac{N_{B}}{H_{B}}\left(\frac{2 N_{E S}}{4 N_{B} / H_{B}}-1\right), \\
g_{R S} & =a_{R S} \frac{D_{R S}}{A_{R S} H_{B}}\left(\frac{2 N_{R S}}{D_{R S} /\left(A_{R S} H_{B}\right)}-1\right),
\end{aligned}
$$

where $\mathrm{N}_{\mathrm{X}}$ is the carrier density in each state, $\mathrm{S}_{\mathrm{GS}}$ is the photon density in the GS, and $\xi$ denotes the gain compression factor. Since the laser emission is resonant with the GS transition $\left(\omega_{L S}^{0}=\omega_{G S}\right)$, the ES and the RS contributions to the real part of the complex gain are negligible (see Fig. 1); therefore, we get the gain of the laser $\operatorname{Re}\left[\tilde{G}\left(\omega_{G S}\right)\right] \approx \Gamma_{P} v_{g} g_{G S}$. In contrast, the imaginary part of the complex gain decays slowly for offresonant frequencies. Therefore, the ES and the RS do influence the refractive index change of GS. The frequency shifts of the laser field induced by carrier populations in the ES and $\mathrm{RS}$ are given respectively by

$$
\begin{aligned}
& \Delta \omega_{N}^{E S}=\frac{1}{2} \Gamma_{P} v_{g} g_{E S} F_{E S}^{G S}, \\
& \Delta \omega_{N}^{R S}=\frac{1}{2} \Gamma_{P} v_{g} g_{R S} F_{R S}^{G S},
\end{aligned}
$$

with coefficients

$$
F_{E S, R S}^{G S}=\frac{\omega_{G S}}{\omega_{E S, R S}} \frac{\left(\omega_{E S, R S}-\omega_{G S}\right) T_{D}}{1+\left(\omega_{E S, R S}-\omega_{G S}\right)^{2} T_{D}^{2}},
$$

where $T_{D}$ is the dephasing time. From Eq. (3), the resonant GS does not contribute to the refractive index change, which is the case when the laser is operated at the gain peak together with a symmetric gain distribution. Nevertheless, the asymmetric QD size dispersion induces a finite $\alpha$-factor $\alpha_{H}^{G S}$ in the GS, and the corresponding frequency shift can be expressed by the relation: ${ }^{20}$

$$
\Delta \omega_{N}^{G S}=\frac{1}{2} \Gamma_{P} v_{g} g_{G S} \alpha_{H}^{G S} .
$$

Employing Eqs. (4)-(8), the complex electric field (2) is reexpressed as

$$
\begin{aligned}
\frac{d E(t)}{d t}= & \frac{1}{2}\left(\Gamma_{P} v_{g} g_{G S}-\frac{1}{\tau_{P}}\right) E(t) \\
& +j\left(\Delta \omega_{N}^{G S}+\Delta \omega_{N}^{E S}+\Delta \omega_{N}^{R S}\right) E(t) .
\end{aligned}
$$

Under carrier injection, the frequency of the laser field becomes $\omega_{L S}=\omega_{L S}^{0}+\Delta \omega_{N}^{L S}$, with the frequency shift of the laser field $\Delta \omega_{N}^{L S}=\Delta \omega_{N}^{G S}+\Delta \omega_{N}^{E S}+\Delta \omega_{N}^{R S}$. Through the relationship $E(t)=\sqrt{S(t) V / \Gamma_{P}} \mathrm{e}^{j \phi(t)}$, the photon density $S(t)$ and the phase $\phi(t)$ can be separately described. Taking into account the carrier dynamics in QD lasers, the laser system is finally described by:

$$
\begin{gathered}
\frac{d N_{R S}}{d t}=\frac{I}{q V}+\frac{N_{E S}}{\tau_{R S}^{E S}}-\frac{N_{R S}}{\tau_{E S}^{R S}}\left(1-\rho_{E S}\right)-\frac{N_{R S}}{\tau_{R S}^{s p o n}} \\
\frac{d N_{E S}}{d t}=\left(\frac{N_{R S}}{\tau_{E S}^{R S}}+\frac{N_{G S}}{\tau_{E S}^{G S}}\right)\left(1-\rho_{E S}\right)-\frac{N_{E S}}{\tau_{G S}^{E S}}\left(1-\rho_{G S}\right) \\
\quad-\frac{N_{E S}}{\tau_{R S}^{E S}}-\frac{N_{E S}}{\tau_{E S}^{s p o n}}, \\
\frac{d N_{G S}}{d t}=\frac{N_{E S}}{\tau_{G S}^{E S}}\left(1-\rho_{G S}\right)-\frac{N_{G S}}{\tau_{E S}^{G S}}\left(1-\rho_{E S}\right)-v_{g} g_{G S} S_{G S}-\frac{N_{G S}}{\tau_{G S}^{s p o n}} \\
\frac{d S_{G S}}{d t}=\left(\Gamma_{p} v_{g} g_{G S}-\frac{1}{\tau_{P}}\right) S_{G S}+\beta_{S P} \frac{N_{G S}}{\tau_{G S}^{s p o n}}, \\
\frac{d \phi}{d t}=\Delta \omega_{N}^{G S}+\Delta \omega_{N}^{E S}+\Delta \omega_{N}^{R S},
\end{gathered}
$$

where $\tau_{G S}^{\text {spon }}$ is the spontaneous emission time and $\beta_{S P}$ is the spontaneous emission factor. $\rho_{G S, E S}$ is the carrier occupation probability of each localized state. Carriers in the RS are scattered into the dots through the phonon-assisted and Auger-assisted processes. ${ }^{21}$ The latter one makes the scattering rates nonlinearly dependent on the carrier density in the $\mathrm{RS}$. However, for the sake of simplicity, the carrier capture time $\tau_{E S}^{R S}$ and the relaxation time $\tau_{G S}^{E S}$ are both treated as constants in this work. On the other hand, the carrier-escape times $\left(\tau_{R S}^{E S}, \tau_{E S}^{G S}\right)$ are governed by the Fermi distribution for a quasi-thermal equilibrium system. ${ }^{18}$ For semiconductor lasers operating under small-signal modulation with frequency $\omega$, the bias current change $\delta I$ induces variations of the carrier densities $\delta N_{X}$, the photon density $\delta S_{G S}$ and the phase $\delta \phi$. Based on the small-signal analysis of the differential rate equations, ${ }^{22}$ the $\alpha$-factor of the QD laser is described as

$$
\begin{aligned}
\alpha_{H, Q D}^{G S}(\omega) & =\frac{2}{\Gamma_{P} v_{g}} \frac{\delta\left[\Delta \omega_{N}^{L S}(N)\right]}{\delta g_{G S}(N)} \\
& \equiv \alpha_{H}^{G S}+\frac{1}{2} F_{E S} \frac{a_{E S} \delta N_{E S}}{a \delta N_{G S}}+2 F_{R S} \frac{a_{R S} \delta N_{R S}}{a \delta N_{G S}}
\end{aligned}
$$

where $a=\partial g_{G S} / \partial N_{G S}$. Following the definition in Eq. (1), it is noted that only the carrier contribution $(\delta N)$ is included in the above equation, while the photon contribution $(\delta S)$ is excluded. In the following, it will be shown that the $\alpha$-factor in QD lasers does depend on the modulation frequency, and that the conventional value is given by the minimum as $\alpha_{H, Q D}^{G S}=\min \left\{\alpha_{H, Q D}^{G S}(\omega)\right\} .^{23}$ 
Over the last decades, various techniques have been proposed for the measurement of the $\alpha$-factor. In this work, we employ the widely used "FM/AM" technique for the abovethreshold and the "amplified spontaneous emission (ASE)" method for the below-threshold analyses, respectively. ${ }^{1}$ The
"FM/AM" technique relies on the direct current modulation of the laser, which generates both the optical frequency (FM) and amplitude (AM) modulations. With respect to the linearized rate equations, the ratio of the FM-to-AM index is derived as

$$
\begin{aligned}
2 \frac{\beta(\omega)}{m(\omega)}= & 2 \frac{\delta \omega_{L S} / \omega}{\delta S_{G S} / S_{G S}} \\
\equiv & \frac{j \omega+\left(1 / \tau_{P}-\Gamma_{P} v_{g} g_{G S}+\Gamma_{p} v_{g} a_{P} S_{G S}\right)}{j \omega} \\
& \times\left[\alpha_{H}^{G S}\left(1-\frac{a_{P} \delta S_{G S}}{a \delta N_{G S}}\right)+\frac{1}{2} F_{E S} \frac{a_{E S} \delta N_{E S}}{a \delta N_{G S}}+2 F_{R S} \frac{a_{R S} \delta N_{R S}}{a \delta N_{G S}}\right],
\end{aligned}
$$

where the relation $\delta \omega_{L S}=j \omega \delta \phi$ is used in the above derivation and $a_{p}=-\partial g_{G S} / \partial S_{G S}$ considering the gain compression effect. In this approach, the laser's $\alpha$-factor is extracted through the formula $\alpha_{H, Q D}^{F M / A M}=\min \{2 \beta(\omega) / m(\omega)\}$.

For semiconductor lasers operating below threshold, the "ASE" method relies on the direct measurement of the optical spectra of ASE in the laser cavity. Tuning the pump current slightly step by step $(\Delta I)$, the gain change can be extracted by the Hakki-Paoli method and the wavelength variation can be directly recorded using an optical spectrum analyzer. Correspondingly, the below-threshold $\alpha$-factor is calculated as

$$
\alpha_{H, Q D}^{A S E}=\alpha_{H}^{G S}+\frac{1}{2} F_{E S}^{G S} \frac{a_{E S}}{a_{G S}} \frac{\Delta N_{E S}}{\Delta N_{G S}}+2 F_{R S}^{G S} \frac{a_{R S}}{a_{G S}} \frac{\Delta N_{R S}}{\Delta N_{G S}} .
$$

The laser under study is based on the $\operatorname{InAs} / \operatorname{InP}(311 \mathrm{~B})$ QD structure operating at room temperature, ${ }^{8,24-26}$ and the laser parameters used in the simulation are listed in Table I.

TABLE I. QD material and laser parameters.

\begin{tabular}{lcc}
\hline \hline Symbol & Description & Value \\
\hline $\mathrm{L}$ & Active region length & $5 \times 10^{-2} \mathrm{~cm}$ \\
$\mathrm{~W}$ & Active region width & $4 \times 10^{-4} \mathrm{~cm}$ \\
$\mathrm{R}_{1}=\mathrm{R}_{2}$ & Mirror reflectivity & 0.32 \\
$\mathrm{n}_{\mathrm{r}}$ & Refractive index & 3.5 \\
$\alpha_{i}$ & Internal modal loss & $6 \mathrm{~cm}^{-1}$ \\
$\mathrm{~N}_{\mathrm{B}}$ & Dot density & $10 \times 10^{10} \mathrm{~cm}^{-2}$ \\
$\mathrm{H}_{\mathrm{B}}$ & Dot height & $5 \times 10^{-7} \mathrm{~cm}$ \\
$\Gamma_{p}$ & Optical confinement factor & 0.06 \\
$\beta_{S P}$ & Spontaneous emission factor & $1 \times 10^{-4}$ \\
$\mathrm{E}_{\mathrm{GS}}$ & GS transition energy & $0.82 \mathrm{eV}$ \\
$\mathrm{E}_{\mathrm{ES}}$ & ES transition energy & $0.87 \mathrm{eV}$ \\
$\mathrm{E}_{\mathrm{WL}}$ & WL transition energy & $0.97 \mathrm{eV}$ \\
$\tau_{E S}^{W L}$ & Capture time from WL to ES & $6.3 \mathrm{ps}$ \\
$\tau_{G S}^{E S}$ & Relaxation time from ES to GS & $2.9 \mathrm{ps}$ \\
$\mathrm{a}_{\mathrm{GS}}$ & GS differential gain & $5 \times 10^{-15} \mathrm{~cm}^{2}$ \\
$\mathrm{a}_{\mathrm{ES}}$ & ES differential gain & $10 \times 10^{-15} \mathrm{~cm}^{2}$ \\
$\mathrm{a}_{\mathrm{RS}}$ & RS differential gain & $2.5 \times 10^{-15} \mathrm{~cm}^{2}$ \\
$\xi$ & Gain compression factor & $2 \times 10^{-16} \mathrm{~cm}^{3}$ \\
$\alpha_{H}^{G S}$ & GS induced $\alpha$-factor & 0.5 \\
$\mathrm{~T}_{\mathrm{D}}$ & Dephasing time & $0.1 \mathrm{ps}$ \\
\hline \hline
\end{tabular}

It is noted that the carrier occupation in the GS has a small contribution to the $\alpha$-factor (less than 1), ${ }^{27}$ hence we assume the value $\alpha_{H}^{G S}=0.5$ in the simulation. Figure 2 depicts the carrier density variations in the three states under small signal modulation. For low frequencies smaller than $0.1 \mathrm{GHz}$, all the carrier density variations remain almost constant, but the variations of the $\mathrm{ES}\left(\delta \mathrm{N}_{\mathrm{ES}}\right)$ and $\mathrm{RS}\left(\delta \mathrm{N}_{\mathrm{RS}}\right)$ populations are $15 \mathrm{~dB}$ larger than that of the $\mathrm{GS}\left(\delta \mathrm{N}_{\mathrm{GS}}\right)$ one. The small variation of the GS carrier population is associated with the gain clamping above threshold. Both $\delta \mathrm{N}_{\mathrm{GS}}$ and $\delta \mathrm{N}_{\mathrm{ES}}$ exhibit resonances around $7 \mathrm{GHz}$. Beyond the resonance frequency, $\delta \mathrm{N}_{\mathrm{GS}}$ decays faster than $\delta \mathrm{N}_{\mathrm{ES}}$ and $\delta \mathrm{N}_{\mathrm{RS}}$. These features significantly impact the behaviour of the $\alpha$-factor as described in Eq. (15). Fig. 3 compares the difference between the $\alpha$-factor $\alpha_{H, Q D}^{G S}(\omega)$ and the ratio $2 \beta(\omega) / m(\omega)$ as a function of the modulation frequency. At low frequencies smaller than $0.1 \mathrm{GHz}$, there is a large discrepancy between the two parameters. As expected, $2 \beta(\omega) / m(\omega)$ exhibits large values due to the gain compression and the large carrier variations in the ES and in the RS. Nevertheless, $\alpha_{H, Q D}^{G S}(\omega)$ remains constant. Increasing the modulation frequency beyond several $\mathrm{GHz}$, the two values of both parameters decrease down to a plateau, which gives the conventional $\alpha$-factor indicated

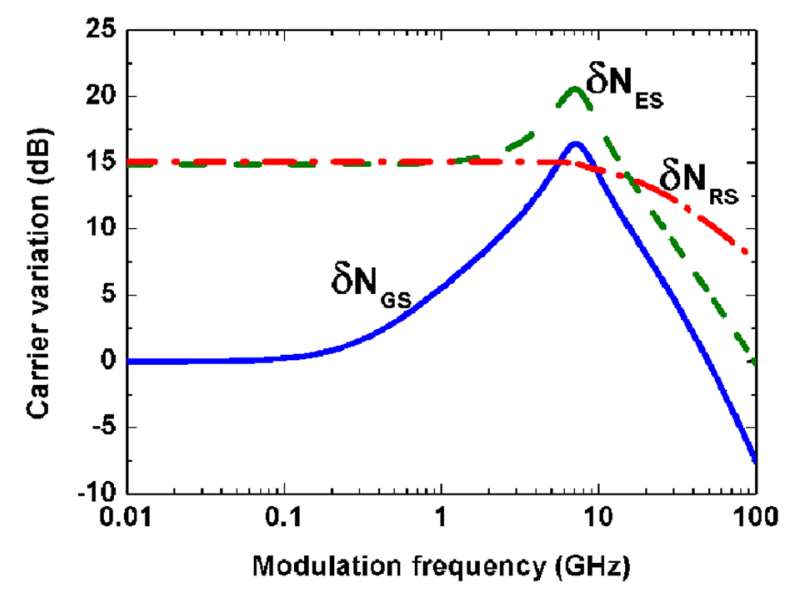

FIG. 2. Small-signal carrier density variations in the GS (solid line), ES (dashed line), and RS (dash-dot line) versus modulation frequency. The bias current is $\mathrm{I}=1.2 \times \mathrm{I}_{\mathrm{th}}$, with the threshold current $\mathrm{I}_{\mathrm{th}}=49 \mathrm{~mA}$. The carrier variation is normalized to the value $\delta \mathrm{N}_{\mathrm{GS}}$ of $0.01 \mathrm{GHz}$. 


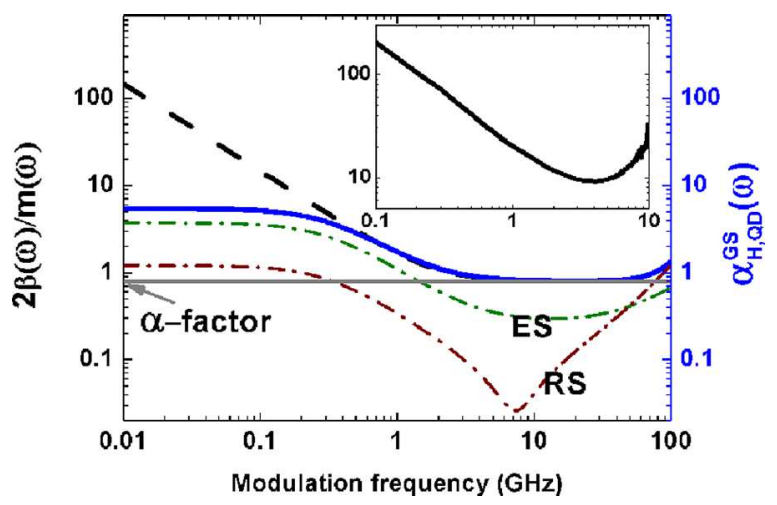

FIG. 3. Modulation-frequency dependence of the FM-to-AM ratio (dash) and of the $\alpha$-factor (thick solid). The minimum level indicated by the horizontal line gives the laser's conventional $\alpha$-factor. The thin dash-dotted curve represents the sole contribution of the ES or the RS to the $\alpha$-factor, respectively. The inset shows an experimental curve of the FM-to-AM ratio for a QD laser.

by the horizontal line. As can be seen, $\alpha_{H, O D}^{F M / A M}$ is almost the same as $\alpha_{H, Q D}^{G S}$, which indicates that the FM/AM method is a reliable technique for the measurement of QD laser's $\alpha$-factor. Further increase of the modulation frequency raises again both the two values as observed experimentally in a QD laser (inset of Fig. 3). It is emphasized that such a situation is not encountered in QW lasers. ${ }^{28}$ This behavior is attributed to the different decay rates (versus modulation frequency) of carrier variations in each state as shown in Fig. 2. In addition, Fig. 3 (dash-dotted curves) shows that the ES contributes more to the $\alpha$-factor $\alpha_{H, Q D}^{G S}$ than the RS due to the smaller energy separation.

Based on the ASE and the FM/AM methods, Fig. 4 illustrates the $\alpha$-factor as a function of the normalized pump current $\mathrm{I} / \mathrm{I}_{\mathrm{th}}$. Below threshold, carrier populations in both the resonant and off-resonant states increase with the pump current. In consequence, the $\alpha$-factor increases nonlinearly. Above threshold, the carrier population in the GS is clamped, while the off-resonant state populations keep increasing. Thus, the $\alpha$-factor varies almost linearly above threshold as usually measured in experiments. ${ }^{8,29}$ At threshold, the $\alpha$-factor extracted from the ASE method is similar to that using the FM/AM technique. In addition, the $\alpha$-factor is

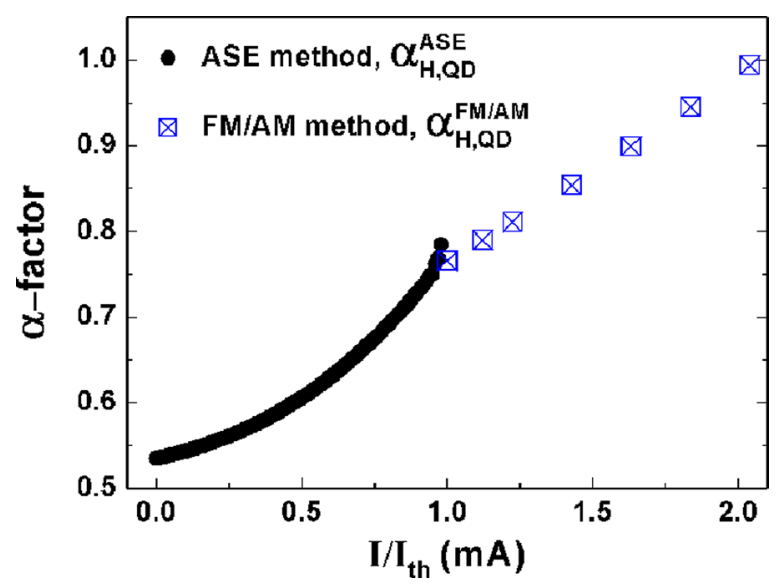

FIG. 4. $\alpha$-factor as a function of the normalized bias current $\mathrm{I} / \mathrm{I}_{\mathrm{th}}$.

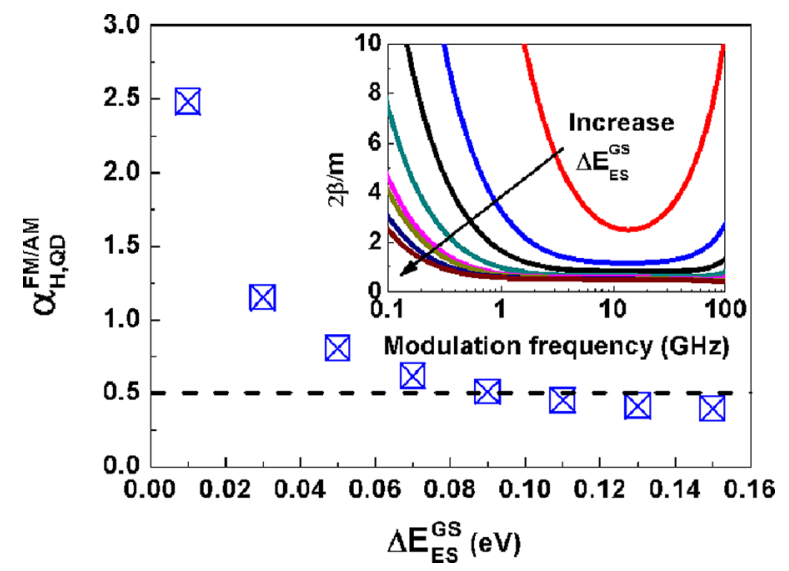

FIG. 5. $\alpha_{H, Q D}^{F M / A M}$ dependence on the GS-ES separation $\Delta E_{E S}^{G S}$ using Eq. (16). The GS transition energy is $\mathrm{E}_{\mathrm{GS}}=0.82 \mathrm{eV}$, and the GS-RS separation is kept as $\Delta E_{R S}^{G S}=3 \times \Delta E_{E S}^{G S}$. Inset shows the corresponding dependence of $2 \beta / \mathrm{m}$ on the modulation frequency. The simulated range is from $10 \mathrm{meV}$ up to $150 \mathrm{meV}$.

larger than the sole GS-induced value of $\alpha_{H}^{G S}=0.5$ both below and above threshold, which means the off-resonant ES and RS contribute to the increase of the $\alpha$-factor in the QD laser. This is explained by the fact that the coefficients $F_{E S}^{G S}$ and $F_{R S}^{G S}$ are both positive since the ES and RS have higher energies than the GS (see Eq. (7)).

In addition, Eq. (7) points out that the $\alpha$-factor coefficients $F_{E S . R S}^{G S}$ strongly rely on the energy separation between the GS and the off-resonant states. Figure 5 depicts the $\alpha$-factor dependence on the GS-ES separation $\Delta E_{E S}^{G S}$, where the GS-RS separation is kept as $\Delta E_{R S}^{G S}=3 \times \Delta E_{E S}^{G S}$. It is noted that the carrier capture and relaxation times are fixed as shown in Table I, since the carrier scattering rates are weakly dependent on the energy separation. ${ }^{30}$ For a small separation $\Delta E_{E S}^{G S}=0.01 \mathrm{eV}$, the laser exhibits a large $\alpha$-factor with a value of 2.5 due to the contribution of carrier populations in the ES and the RS, which is 5-fold higher than $\alpha_{H}^{G S}$. Enlarging the energy separation reduces the value of $\alpha$-factor. ${ }^{31}$ The value can be even slightly smaller than $\alpha_{H}^{G S}$ due to the gain compression as shown in Eq. (15). The inset of Fig. 5 presents the corresponding variation of $2 \beta / \mathrm{m}$ as a function of the modulation frequency. It shows that QD lasers of larger energy separation not only exhibit a smaller $\alpha$-factor, but also a lower FM-to-AM index ratio over all the low and high frequencies. Especially, large energy separation significantly suppresses the re-increase of $2 \beta / m$ at high modulation frequencies due to the decoupling between the GS and the off-resonant states. Therefore, strongly confined QDs are more favorable to realize low chirp laser devices with small $\alpha$-factor.

In conclusion, a theoretical model for studying the $\alpha$-factor of QD lasers is proposed. Unambiguous definitions of $\alpha$-factor in relation with experimental techniques are described. Employing this model, simulations show that the $\alpha$-factor value strongly depends on the energy separation between the lasing GS and the off-resonant states. There is a potential to reduce the QD laser's $\alpha$-factor through enlarging that energy separation in the band energy engineering. This work also explains the wide range of $\alpha$-factor values observed in experiments and opens the way for a better control of the $\alpha$-factor in semiconductor QD lasers. 
The authors would like to thank Dr. Philip Poole from the National Research Council, Canada, for providing the QD lasers, and Dr. Jean-Guy Provost from III-V Lab, France for assisting experiments. The authors thank Dr. M. Gioannini, Professor I. Montrosset from Politecnico di Torino, Italy, and Dr. K. Lüdge, B. Lingnau from Technische Universität Berlin, Germany, for helpful discussions. C. Wang's work was supported by China Scholarship Council. M. Osiński was supported by NanoDesign and Télécom ParisTech. F. Grillot's work was supported by Partenariat Hubert Curien under Grant No. 30794RC (Campus France/DAAD).

${ }^{1}$ M. Osiński and J. Buus, IEEE J. Quantum Electron. QE-23, 9 (1987).

${ }^{2}$ S. Wieczorek, B. Krauskopf, T. B. Simpson, and D. Lenstra, Phys. Rep. 416, 1 (2005).

${ }^{3}$ B. Globisch, C. Otto, E. Schöll, and K. Lüdge, Phys. Rev. E 86, 046201 (2012).

${ }^{4}$ G. P. Agrawal, IEEE Photonics Technol. Lett. 1, 212 (1989).

${ }^{5}$ L. A. Coldren and S. W. Corzine, Diode Lasers and Photonic Integrated Circuits (Wiley, 2012).

${ }^{6}$ D. Bimberg, M. Grundmann, and N. N. Ledentsov, Quantum Dot Heterostructures (Wiley, 1999).

${ }^{7}$ T. C. Newell, D. J. Bossert, A. Stintz, B. Fuchs, K. J. Malloy, and L. F. Lester, IEEE Photon. Tech. Lett. 11, 1527 (1999).

${ }^{8}$ A. Martinez, K. Merghem, S. Bouchoule, G. Moreau, A. Ramdane, J. G. Provost, F. Alexandre, F. Grillot, O. Dehaese, R. Piron, and S. Loualiche, Appl. Phys. Lett. 93, 021101 (2008).

${ }^{9}$ B. Dagens, A. Markus, J. X. Chen, J. G. Provost, D. Make, O. LeGoueziou, J. Landreau, A. Foire, and B. Thedrez, Electron. Lett. 41, 323 (2005).

${ }^{10}$ M. Gioannini, A. Sevega, and I. Montrosset, Opt. Quantum Electron. 38, 381 (2006).

${ }^{11}$ B. Lingnau, K. Lüdge, W. W. Chow, and E. Schöll, Phys. Rev. E 86, 065201(R) (2012).
${ }^{12}$ S. Melnik, G. Huyet, and A. V. Uskov, Opt. Express 14, 2950 (2006).

${ }^{13}$ A. V. Uskov, E. P. O'Reilly, D. McPeake, N. N. Ledentsov, D. Bimberg, and G. Huyet, Appl. Phys. Lett. 84, 272 (2004).

${ }^{14}$ S. P. Hegarty, B. Corbett, J. G. McInerney, and G. Huyet, Electron. Lett. 41, 416 (2005).

${ }^{15}$ W. W. Chow and F. Jahnke, Prog. Quantum Electron. 37, 109 (2013).

${ }^{16}$ B. Lingnau, W. W. Chow, E. Schöll, and K. Lüdge, New J. Phys. 15, 093031 (2013).

${ }^{17}$ L. Wang, V. Krapek, F. Ding, F. Horton, A. Schliwa, D. Bimberg, A. Rastelli, and O. G. Schmidt, Phys. Rev. B 80, 085309 (2009).

${ }^{18}$ A. Markus, J. X. Chen, O. Gauthier-Lafaye, J. G. Provost, C. Paranthoën, and A. Foire, IEEE J. Sel. Top. Quantum Electron. 9, 1308 (2003).

${ }^{19}$ C. Wang, B. Lingnau, K. Lüdge, J. Even, and F. Grillot, IEEE J. Quantum Electron. 50, 723 (2014).

${ }^{20}$ K. Lüdge and H. G. Schuster, Nonlinear Laser Dynamics: From Quantum Dots to Cryptography (Wiley, 2011).

${ }^{21}$ B. Ohnesorge, M. Albrecht, J. Oshinowo, A. Forchel, and Y. Arakawa, Phys. Rev. B 54, 11532 (1996).

${ }^{22}$ C. wang, F. Grillot, and J. Even, IEEE J. Quantum Electron. 48, 1144 (2012).

${ }^{23}$ B. Lingnau, W. W. Chow, and K. Lüdge, Opt. Express 22, 4867 (2014).

${ }^{24}$ C. Cornet, C. Labbé, H. Folliot, N. Bertru, O. Dehaese, J. Even, A. Le Corre, C. Paranthoën, C. Platz, and S. Loualiche, Appl. Phys. Lett. 85, 5685 (2004).

${ }^{25}$ C. Platz, C. Paranthoën, P. Caroff, N. Bertru, C. Labbé, J. Even, O. Dehaese, H. Folliot, A. Le Corre, S. Loualiche, G. Moreau, J. C. Simon, and A. Ramdane, "Comparison of InAs quantum dot lasers emitting at $1.55 \mathrm{~m}$ under optical and electrical injection," Semicond. Sci. Technol. 20, 459 (2005).

${ }^{26}$ N. F. Massé, E. Homeyer, A. R. Adams, S. J. Sweeney, O. R. Piron, F. Grillot, and S. Loualiche, Appl. Phys. Lett. 91, 131113 (2007).

${ }^{27}$ Z. Mi and P. Bhattacharya, IEEE J. Quantum Electron. 43, 363 (2007).

${ }^{28}$ J. G. Provost and F. Grillot, IEEE Photonics J. 3, 476 (2011).

${ }^{29} \mathrm{M}$. Gioannini and I. Montrosset, IEEE J. Quantum Electron. 43, 941 (2007).

${ }^{30}$ K. Schuh, P. Gartner, and F. Jahnke, Phys. Rev. B 87, 035301 (2013).

${ }^{31}$ J. Molina, H. H. Nilsson, J. Z. Zhang, and I. Galbraith, IEEE J. Quantum Electron. 42, 986 (2006). 